\title{
LA REFORMA ENERGÉTICA (2013-2014) A LA LUZ DE LA NUEVA LEGISLACIÓN SOBRE LOS IMPACTOS SOCIALES DE LOS PROYECTOS
}

\author{
THE ENERGY REFORM (2013-2014) IN LIGHT \\ OF THE NEW LEGISLATION ON THE SOCIAL \\ IMPACTS OF PROJEGTS
}

\author{
LA RÉFORME ÉNERGÉTIQUE (2013-2014) À LA \\ LUMIÈRE DES NOUVELLES LOIS SUR LES EFFETS \\ SOCIAUX DES GRANDS PROJETS
}

\author{
ISABELLE Rousseau \\ El Colegio de México \\ irouss@colmex.mx
}

Resumen: En el marco de la reforma energética, en 2014, las leyes de Hidrocarburos y de la Industria Eléctrica incluyeron herramientas para prevenir, mitigar o reparar los posibles daños que la presencia de la infraestructura de los megaproyectos del sector puede causar en las comunidades o los pueblos. Este esfuerzo de regulación de la gestión social de los proyectos responde a la promoción del desarrollo sostenible del sector, que combina tanto la seguridad energética del país (disponibilidad de productos energéticos a precios adecuados) como la gobernanza de los proyectos (otorgar certeza jurídica a las inversiones públicas o privadas, pero también proteger los derechos de las comunidades). Se analizarán aquí tanto las aportaciones de las nuevas normas como sus posibles deficiencias.

Palabras clave: conflictos sociales; regulación de la gestión social; proyectos energéticos; seguridad energética; gobernabilidad.

ABSTRACT: In the context of the 2014 energy reform, the laws on the hydrocarbon and the electricity sector included tools for preventing, mitigating or repairing the possible damage that the infrastructure of mega-projects relating to this sector may cause to communities. This effort to regulate the social impact of projects responds to the promotion of sustainable development by the sector, combining the country's energy security (availability of energy prod- 
ucts at accessible prices) with governance of the projects (granting legal certainty to public and private investments, while protecting the rights of communities). This paper will analyze both the new regulations and their potential shortcomings.

Keywords: social conflicts, social impact and regulation, energy projects, energy security, governance.

\section{Traducción de Gonzalo Celorio Morayta}

Résumé: Dans le cadre de la réforme énergétique de 2014, les lois concernant les hydrocarbures et l'industrie électrique ont créé des outils pour éviter, atténuer ou réparer les dommages que l'infrastructure nécessaire aux mégaprojets dans ce secteur pourrait causer aux communautés ou aux villages. L'effort pour réglementer la gestion sociale des projets poursuit un développement soutenable qui puisse assurer la sécurité du pays en matière d'énergie (c'est à dire la disponibilité énergétique à des prix raisonnables) aussi bien que la gouvernance des projets (en offrant une certitude juridique aux investissements publics ou privés, tout en protégeant les droits des communautés). On analyse ici les avantages de ces nouvelles normes, ainsi que les inconvénients qu'elles risquent d'avoir.

Mots clefs: conflits sociaux; régulation, gestion sociale; projets énergétiques; sécurité énergétique; gouvernance.

Traducción de BERNARDo Mabire

Fecha de recepción: junio de 2019

Fecha de aceptación: noviembre de 2019 
$\mathrm{L}$ as reformas a los artículos 25, 27 y 28 de la Constitución de los Estados Unidos Mexicanos que fueron aprobadas a finales de diciembre de $2013^{1}$ y las leyes reglamentarias emitidas el año siguiente ${ }^{2}$ representaron un parteaguas en la historia del sector energético mexicano. La apertura de par en par de toda la cadena de valor de las dos industrias (eléctrica e hidrocarburos) a la inversión privada significó un cambio de paradigma que no tuvo equivalencia en un sector que creció a la sombra de procesos de nacionalización dotados de un alto valor simbólico (1938, para el petróleo; 1960, para la electricidad). Esta reforma significó el final de los dos monopolios de Estado -Petróleos Mexicanos (Pemex) y Comisión Federal de Electricidad (CFE) - y la creación de una industria petrolera y eléctrica que no se reduzca a esas dos empresas como únicos oferentes. Sin embargo, para evitar toda confusión, se especificó, en el caso de los hidrocarburos, que las reservas seguían perteneciendo de manera exclusiva a la nación.

La reforma puso el punto final a una larga serie de transformaciones institucionales de menor alcance que, desde finales de los años ochenta, las diversas administraciones habían impulsado en aras de modernizar el sector y ponerlo a tono con el proyecto financiero y económico de corte liberal que empezaba a predominar en el país. ${ }^{3}$ Sin embargo, por distintas razones, no tocaron las piezas fundamentales del sistema energético nacional. Eran más bien cambios cosméticos que, sin

${ }^{1}$ La reforma constitucional fue aprobada el 12 de diciembre y fue publicada posteriormente. Véase Decreto por el que se reforman y adicionan diversas disposiciones de la Constitución Política de los Estados Unidos Mexicanos, en Materia de Energía, Diario Oficial de la Federación, 22 de diciembre de 2013, http://www.dof.gob.mx/nota_detalle.php?codi go $=5327463 \&$ fecha $=20 / 12 / 2013$

${ }^{2}$ Las leyes secundarias fueron aprobadas el 12 de agosto de 2014. Se introdujeron nueve leyes y se modificaron catorce apartados de leyes en existencia.

${ }^{3}$ También se trataba de ponerlo a tono con los principios rectores de estas dos industrias a nivel internacional. 
embargo, no dejaban de tener efectos sobre el funcionamiento del sector. ${ }^{4}$ Cortando de manera tajante con este gradualismo, la reforma energética creó mecanismos e instituciones nuevos y ajustó otras piezas para propiciar la apertura de un sector hasta ese momento protegido. En este tenor, estableció dos criterios a fin de orientar objetivos, estrategias y operaciones en ambas industrias y empresas estatales: 1) la creación de valor (la búsqueda para maximizar el valor de cualquier actividad) en un ámbito de competitividad económica y comercial, 2) el desarrollo sostenible del sector (que genere las condiciones de su propia continuidad y provea un crecimiento económico beneficioso sin comprometer las necesidades de las futuras generaciones). ${ }^{5}$ Basada en el segundo principio, la reforma va a otorgar un papel importante a los aspectos sociales y ambientales asociados a la presencia de infraestructura de los proyectos energéticos en las comunidades. ${ }^{6}$ Hasta el momento, ambos aspectos no estaban

${ }^{4}$ Las administraciones anteriores buscaron reorganizar el andamiaje institucional del sector para simular un ámbito de mercado en industrias operando con una lógica de monopolio de Estado. Esto respondió a razones de índole político-electoral: preservar la estabilidad política y social en momentos en que los fundamentos del viejo régimen venían siendo atacados con la apertura de la economía, que encumbró la firma del Tratado de Libre Comercio de América del Norte, así como con la transición democrática. Sin contar con que a partir del 2000 predominaron los "gobiernos divididos", es decir, que ningún partido tenía una mayoría suficiente para asegurar cambios constitucionales.

${ }_{5}$ Pemex "tiene como fin el desarrollo de actividades empresariales, económicas, industriales y comerciales en términos de su objeto, generando valor económico y rentabilidad para el Estado mexicano como su propietario, así como actuar de manera transparente, honesta, eficiente, con sentido de equidad y responsabilidad social y ambiental, y procurar el mejoramiento de la productividad para maximizar la renta petrolera del Estado y contribuir con ello al desarrollo nacional". Ley de Petróleos Mexicanos, 2014, Diario Oficial de la Federación, 11 de agosto de 2014, artículo 4, https:/ / www.dof.gob.mx/nota_detalle.php?codigo=5355990\& fecha $=11 /$ $08 / 2014$

${ }^{6}$ Por su lado, la Comisión Federal de Electricidad tiene como objetivo "el desarrollo de actividades empresariales, económicas, industriales y co- 
vinculados a la creación de valor económico pero, además, solía considerárseles como obstáculos a la maximización del valor. En este nuevo contexto, la integración de lo social en la reforma energética de 2014 busca otorgar certeza jurídica a las partes, respetar los derechos humanos y generar un desarrollo acorde con los Objetivos de Desarrollo Sostenible (oDs) de la Organización de las Naciones Unidas (ONU). ${ }^{7}$ En otros términos, la nueva legislación, enriquecida con una nueva regulación social, debería ayudar a que las empresas (públicas o privadas) obtuvieran la famosa "licencia social" para operar. Sin embargo, a la fecha, la implementación de la reforma no ha dado resultados del todo satisfactorios. Los conflictos no han disminuido de manera notable y el entendimiento entre las partes sigue siendo muy complicado.

En dicho contexto, este artículo se enfoca en estudiar la creación del dispositivo institucional establecido para atender los potenciales conflictos vinculados con la presencia de los proyectos de hidrocarburos o eléctricos en los pueblos y comunidades del país (Ley de Hidrocarburos y Ley de la Industria Eléctrica, de 2014), así como sus aportaciones y limitantes. La elección de este aspecto se debe a que el tema de

merciales en términos de su objeto, generando valor económico y rentabilidad para el Estado mexicano como su propietario. En la ejecución de su objeto, la CFE deberá actuar de manera transparente, honesta, eficiente, con sentido de equidad, y responsabilidad social y ambiental, procurando el mejoramiento de la productividad con sustentabilidad para minimizar los costos de la industria eléctrica en beneficio de la población y contribuir con ello al desarrollo nacional". Ley de la Comisión Federal de Electricidad, 2014, Diario Oficial de la Federación, 11 de agosto de 2014, artículo 4, https://www.dof.gob.mx/nota_detalle.php?codigo=5355990\& fecha $=11 / 08 / 2014$

${ }^{7}$ En la Cumbre para el Desarrollo Sostenible, en septiembre de 2015, los Estados miembros de la ONU aprobaron la Agenda 2030 para el Desarrollo Sostenible, que incluye un conjunto de 17 Objetivos de Desarrollo Sostenible (oDs) para poner fin a la pobreza, luchar contra la desigualdad y la injusticia, y hacer frente al cambio climático, basados en los Objetivos de Desarrollo del Milenio. Vease Naciones Unidas, Objetivos de Desarrollo Sostenible, 2015, https://www.un.org/sustainabledevelopment/es/ob jetivos-de-desarrollo-sostenible/ 
la regulación de los impactos sociales -a pesar de constituir una aportación institucional y normativa muy importante e innovadora en el país- no se ha beneficiado de la misma visibilidad mediática que el resto de la reforma energética. Se ha considerado como una cuestión secundaria por no pertenecer al core business del sector. Esto no deja de ser extraño a sabiendas de que los problemas sociales, así como los ambientales, representan uno de los nudos gordianos en el ramo: suelen frenar o detener el avance de los proyectos y, por lo tanto, ponen en riesgo la seguridad energética del país y la sustentabilidad de los proyectos, así como la gobernanza del sector, además de constituir uno de los ejes fundamentales que articulan la capacidad de promover un mejor desarrollo socioeconómico de las comunidades y un manejo eficiente de los proyectos energéticos. Como estos dos aspectos no suelen ajustarse de manera automática ni armónica en el sector de la energía, era necesario crear una regulación que ofrezca un marco de certidumbre y seguridad jurídica para las partes involucradas.

Nos proponemos aquí presentar el nuevo marco institucional y regulatorio en lo social creado a la luz de la reforma constitucional energética de 2013 y la legislación secundaria correspondiente, emitida en 2014, con la intención de analizar tanto sus aportaciones como sus deficiencias y limitantes. Las preguntas fundamentales que van a guiar este examen son las siguientes: ¿Hasta qué punto esta nueva legislación puede propiciar una gobernabilidad adecuada para la gestión social de los proyectos? ¿Cuáles son las aportaciones de este nuevo marco regulatorio y cuáles son sus limitantes? Finalmente, ¿qué mecanismos podrían propiciar mayor certeza jurídica, atenuar las asimetrías entre los diferentes actores y ofrecer mayor y mejor seguridad y sostenibilidad energética?

Para empezar, se ofrece una revisión de la coyuntura prevalente en cuestiones sociales en el momento de la reforma energética, así como los principales obstáculos a los que se ha enfrentado este cambio regulatorio. 


\section{LA NUEVA LEGISLACIÓN PARA REGULAR LOS IMPACTOS SOCIALES VINCULADOS CON LOS MEGAPROYECTOS ENERGÉTICOS}

Propiciar mayor desarrollo industrial y económico, además de atender la seguridad energética, así como la sustentabilidad del sector, fueron algunos de los objetivos oficiales que justificaban la reforma y las medidas adoptadas en temas de energía. Al alentar el ingreso de promoventes en el sector, fue necesario diseñar mecanismos para prevenir y atender las posibles controversias de impacto socioambiental y regularización de la ocupación superficial de las tierras relacionadas con la presencia de infraestructura de gran calibre, propia de proyectos energéticos estatales o privados en municipios, comunidades y ejidos. Se crearon normas jurídicas que garantizan a los propietarios y usufructuarios de la tierra derechos mínimos y reparaciones justas y equitativas por las probables repercusiones ligadas a la infraestructura de megaproyectos. $\mathrm{Al}$ mismo tiempo, se buscaba garantizar a los promoventes la ausencia de conflictividad social para atraer inversiones. ${ }^{8}$ Se añadieron dos capítulos tanto a la Ley de Hidrocarburos como a la de Industria Eléctrica: uno dedicado al tema de la ocupación superficial y otro al impacto social y la mitigación de sus efectos. Los impactos ambientales no fueron incluidos en las nuevas leyes porque este segmento ya contaba con una regulación, la Manifestación de Impacto Ambiental (MIA), creada en $1996 .{ }^{9}$

¿Cómo surgió esta nueva legislación en el marco de la reforma energética? Si bien ambas industrias habían sido objeto de diagnósticos múltiples desde hace más de quince años, así como de propuestas de cambio, el tema de las externalidades sociales nunca había sido tomado en cuenta.

8 Véase Javier Zenteno et al., "Impacto social y ocupación temporal", Energía a Debate, 2016, núm. 74, p. 62.

${ }^{9}$ La separación entre estas dos legislaciones, como lo veremos, impactará negativamente en la implementación de las herramientas para atender la problemática social. 
Su creación y su diseño institucional fueron la aportación de un pequeño grupo dentro de la Secretaría de Energía (Sener), en el periodo 2012-2014, que se afanó en visibilizar la urgencia de atender una situación potencialmente explosiva. ${ }^{10} \mathrm{El}$ conflicto que acompañó el proyecto Mareña Renovables a partir de 2012 estimuló al equipo de la Subsecretaría de Electricidad, a cargo de Lourdes Melgar, a tomar conciencia de la importancia del tema si se quería abrir de par en par las puertas del sector a la inversión privada. ${ }^{11}$ ¿Cómo atraer a las empresas privadas para alcanzar la seguridad energética y, al mismo tiempo, incluir las demandas de las comunidades y poblaciones en donde se iban a desarrollar los proyectos, en particular, las relativas a posibles daños colaterales? ¿Cómo lograr que las empresas y las instituciones financieras que iban a arriesgar su capital tuvieran certeza sobre la construcción y la operación de los proyectos? En otras palabras, había que resolver la tensión entre diferentes responsabilidades y misiones del Estado: garantizar la seguridad energética (es decir, responder a la demanda), por un lado, y respetar y proteger los derechos de las comunidades y poblaciones donde se iban a desarrollar los proyectos, por el otro. Se trataba de buscar un balance aceptable entre los fines económicos (inversión rentable),

${ }^{10}$ Fue producto de la tenacidad, en particular, de Lourdes Melgar -entonces subsecretaria de Electricidad en la Sener- que tuvo que enfrentar la situación cuando explotó el proyecto eólico Mareña Renovables.

${ }^{11}$ El proyecto Mareña Renovables, que se convirtió luego en Energía Eólica del Sur, pretendía construir una planta de energía eólica de 396 kW en Oaxaca y la infraestructura asociada en el área del istmo de Tehuantepec. El proyecto debía proveer energía a Fomento Económico Mexicano (FEMsA) bajo el marco legal del Programa de Autoabastecimiento. Con una inversión de 850 millones de dólares, el proyecto fue aprobado el 23 de noviembre de 2011 y el contrato, firmado el 23 de febrero de 2012. Sin embargo, el 11 de diciembre de 2015, el juez séptimo de Distrito en el estado de Oaxaca lo suspendió de manera definitiva a raíz de un amparo presentado por 1166 miembros del pueblo indígena binnizá (zapotecos) del istmo de Tehuantepec. 
la seguridad energética a mediano y largo plazo, y la gobernabilidad. ${ }^{12}$

Hasta ese momento, las demandas sociales y las posibles repercusiones para las comunidades habían sido gestionadas de manera parcial y bastante oscura por Pemex y la CFE; en el fondo, las pérdidas en lo que respecta a los cuestiones monetarias y de productividad que esto representaba para ambas paraestatales corrían a cargo del presupuesto federal. Sin embargo, este esquema se veía complicado en el nuevo contexto. Las empresas productivas del Estado, sometidas a una fuerte competencia, y las empresas privadas difícilmente iban a aceptar la absorción de enormes pérdidas, producto de los conflictos sociales. Se constituyó entonces un pequeño equipo -primero en la Subsecretaría de Electricidad y luego en la Subsecretaría de Hidrocarburos- encargado de elaborar una propuesta para incluir el tema en las leyes secundarias. El anteproyecto fue discutido en el poder legislativo. En el marco del Pacto por México, tres partidos se pronunciaron de manera abierta, en particular, sobre el tema de las consultas indígenas. ${ }^{13}$ Tras su aprobación, este equipo

${ }^{12}$ Isabelle Rousseau, "La nueva regulación de la gestión social de los proyectos energéticos en México, seguridad, sustentabilidad y gobernabilidad”, Revista Mexicana de Ciencias Políticas y Sociales (2017), núm. 230, pp. 197-220 .

${ }^{13}$ Por un lado, el Partido Verde Ecologista de México (PVEM), bajo la batuta de la senadora Ninfa Salinas Sada, defendió con vigor la propuesta de la reforma energética y, por ende, de los nuevos artículos tocando los temas de impacto social y consulta indígena. Por otro, el Partido del Trabajo (PT) y el Partido de la Revolución Democrática (PRD) se pronunciaron en contra, alegando que la reforma despojaría a campesinos, ejidatarios, indígenas o afroamericanos de sus propias tierras. Sin embargo, frente al peso del Partido Revolucionario Institucional (PRI), el Partido Acción Nacional (PAN) y el Pvem, las voces opositoras no tuvieron mayoría para anular la inclusión de los temas sociales en ambas leyes (de hidrocarburos y de la industria eléctrica). Véase Diario de los Debates de la Cámara de Senadores del Congreso de los Estados Unidos Mexicanos, LXII Legislatura, año II, tercer periodo extraordinario, vols. II, III, IV y VI. 
diseñó el nuevo marco institucional y las diversas herramientas, para luego ponerlo en marcha.

Las nuevas piezas y reglas relativas a los impactos sociales se construyeron con base en antecedentes legales e institucionales en diversos campos. En efecto, desde los años noventa, y con más vigor a inicios del siglo XxI, se dieron cambios importantes en materia de derechos humanos y de derechos indígenas, así como en materia agraria. Esto propició la aparición de nuevos actores y nuevas demandas que la administración del presidente Ernesto Peña Nieto tuvo que incluir, buscando, por otro lado, respetar las mejores prácticas a nivel internacional en el tema energético. A continuación, se revisarán las transformaciones legales que surgieron en México desde inicios del siglo xxI respecto al tema de derechos humanos.

Los cambios en lo social antes de la reforma: una visión del contexto

\section{En la Constitución}

A raíz de la preparación y luego de la negociación del Tratado de Libre Comercio de América del Norte (TLCAN), a inicios de los años noventa, los derechos humanos y, paralelamente, los derechos de los pueblos indígenas empezaron a adquirir relevancia en México. ${ }^{14}$ El gobierno de Carlos Salinas de

${ }^{14}$ El 6 de junio de 1990, por decreto presidencial, se creó la Comisión Nacional de Derechos Humanos. En un inicio, era un órgano desconcentrado de la Secretaría de Gobernación. Así se pretendía redefinir las obligaciones y responsabilidades del Estado frente al individuo. Sin embargo, fue más que nada un elemento que buscaba proyectar la intención de un cambio de actitud del gobierno mexicano hacia los derechos humanos. En ese momento, dependían directamente de la presidencia de la República. La Comisión Nacional de Derechos Humanos (CNDH) adquiriría posteriormente mayor autonomía y rango constitucional. Isabelle Rousseau, México: ¿Una revolución silenciosa? 1970-1995. Élites gubernamentales y proyecto de modernización, México. Colmex, 2001, p. 361. 
Gortari tenía que ofrecer señales a sus futuros socios comerciales de respeto y garantías a los derechos humanos. Además, con el levantamiento del Ejército Zapatista de Liberación Nacional (EZLN), el 1 de enero de 1994, las exigencias de las comunidades indígenas tomaron fuerza y legitimidad, y las administraciones siguientes se esforzaron en proporcionar mayor atención a sus reclamos. ${ }^{15}$ Se tomaron, entonces, una serie de decisiones que señalan claramente el viraje del Estado mexicano en estas dos cuestiones.

En 1990, el Senado de la República aprobó el Convenio 169 de la Organización Internacional del Trabajo (OIT), que prevé el mecanismo de consulta previa a los indígenas, para aplicarse en $1991 .{ }^{16}$ En un inicio, fue letra muerta; las autoridades mexicanas no observaron la aplicación de las normas contenidas en dicho convenio. Sin embargo, a partir de 2012, la Suprema Corte de Justicia de la Nación las invocó en dos ocasiones: en el caso del Acueducto Independencia, respecto al amparo en revisión 631/2012, y en el caso de Wirikuta. En el segundo, la Comisión Nacional de Derechos Humanos (CNDH) emitió su recomendación 56/2012 sobre la consulta a pueblos y comunidades indígenas en cuanto a la protección del territorio del pueblo wixarika en Wirikuta. ${ }^{17}$

El 21 de mayo de 2003 se constituyó la Comisión Nacional para el Desarrollo de los Pueblos Indígenas (CDI) como órgano descentralizado de la administración pública fede-

${ }^{15}$ El EzLn quería evidenciar que México seguía siendo un país subdesarrollado, contrario a la imagen que el equipo salinista había vendido a Canadá y a Estados Unidos.

${ }^{16}$ El Convenio 169 de la oIt sobre Pueblos Indígenas y Tribales en Países Independientes (1989) establece la obligación, en caso de la existencia de medidas administrativas o legislativas que podrían afectarlos, de consultar a pueblos y comunidades mediante procedimientos apropiados y a través de sus instituciones representativas.

17 Comentarios por escrito (correo electrónico) de Elizabeth Castro, directora adjunta de Ocupación Superficial de la Dirección General de Impactos Sociales y Ocupación Superficial de la Secretaría de Energía (2017). 
ral. Aún más decisiva fue la jurisprudencia que emitió la Corte Interamericana de Derechos Humanos (Corte IDH), en 2009, por el caso Rosendo Radilla Pacheco vs los Estados Unidos Mexicanos. ${ }^{18}$ La sentencia de la Corte obligó a las autoridades a observar el bloque de constitucionalidad: dicta que, además de observar la norma constitucional, es menester acatar también los tratados internacionales que México ha firmado (como el citado Convenio 169 de la OIT) por tener el mismo nivel de importancia legal. ${ }^{19}$ Dicha sentencia llevó a modificar el artículo 1 constitucional, que introdujo el principio pro persona y la denominada interpretación conforme para la aplicación efectiva de los derechos humanos contenidos en la Constitución y en los tratados internacionales. La reforma constitucional de 2011 que incluyó el reconocimiento de los derechos humanos de las personas y estableció garantías para su protección es producto de dichos cambios. En 2012, la Constitución, en su artículo 2, reconoció la composición pluricultural de la nación que, según el Instituto Nacional de Lenguas Indígenas (Inali), se refleja en la existencia de once familias lingüísticas y 364 variantes dialectales.

Por su parte, la preocupación que deben tener las empresas por lo social y los derechos humanos fue catalogada bajo el concepto genérico de "responsabilidad social empresarial

${ }^{18}$ Rosendo Rodilla fue asesinado en Guerrero en los setenta -presuntamente por el gobierno federal, estatal o local-, en tiempos de enfrentamientos entre la guerrilla y el gobierno. Las demandas de su familia no prosperaron durante mucho tiempo; sin embargo, en 2009, la CNDH retomó el caso.

${ }^{19}$ Se ha dicho muchas veces que el bloque de constitucionalidad ha posibilitado la judicialización de muchos proyectos y es probable. El primer caso fue el de Wirikuta, uno de los territorios sagrados de la cosmogonía indígena de los wixarika, que llevó el Centro Mexicano de Derechos Ambientales (CEMDA). Sin embargo, la comunidad perdió el amparo contra la minera canadiense Revolution Resources. Isabelle Rousseau, entrevista con Héctor Garza, 30 de enero de 2017. Sobre Wirikuta, véase Arturo Gutiérrez del Ángel, La peregrinación a Wirikuta: el gran rito de paso de los huicholes. Etnografía de los pueblos indígenas de México, México, INHA / Universidad de Guadalajara, 2002. 
(RSE)", que la iniciativa denominada Principios del Ecuador (PE), lanzada el 4 de junio de 2003, en Washington, EEUU, se encargaría de especificar. En consonancia con el United Nations Global Compact (Pacto Global de Naciones Unidas, instituido el 26 de julio de 2000), los PE establecen que los bancos y las instituciones que financian megaproyectos de infraestructura deberán evaluar el alcance de los riesgos ambientales y sociales asociados a dichos proyectos. Para ello, proporcionan criterios y mecanismos para administrar el riesgo crediticio al determinar, evaluar y gestionar el impacto ambiental y social en proyectos financiados para el desarrollo y la construcción de grandes proyectos de infraestructura e industriales. Obliga a las instituciones financieras firmantes a rechazar préstamos cuando el prestatario no va a poder cumplir con sus respectivas políticas y procedimientos sociales y ambientales. En 2005, otra iniciativa de las Naciones Unidas, los Principios de Inversión Responsable (PIR), reforzó los PE favoreciendo inversiones en los proyectos que incluyan en sus operaciones temas de gobernanza, impacto social e impacto ambiental. Por su parte, las iniciativas institucionales y las experiencias de otros países latinoamericanos en este campo (en particular, Colombia, Ecuador, Perú y Bolivia) influyeron el diseño mexicano.

Finalmente, la reforma constitucional que dio lugar a la Ley Agraria promulgada el 26 de febrero de 1992, en aras de reorientar y dinamizar el desarrollo rural, y combatir la pobreza y la marginación, ${ }^{20}$ repercutiría sobre la ocupación superficial del suelo en el sector energético. A partir del artículo 56 de dicha ley, se buscó dar certidumbre jurídica a la tenencia de la tierra por medio del Programa de Certificación de Derechos Ejidales y Titulación de Solares Urbanos (PROCEDE) ${ }^{21}$

${ }^{20}$ Véase Arturo Warman, "La reforma agraria mexicana: una visión de largo plazo”, Land Reform/Réforme Agraire/Reforma Agraria (2003), núm. 2, pp. 84-94.

21 Se creó, en el PROCEDE, el Registro Agrario Nacional (RAN) al que competen la expedición, registro y control de los certificados de derechos parcelarios y de derechos comunes, así como de los títulos de los solares 
Ese artículo reconoció a las asambleas ejidales y comunales como las autoridades máximas de los núcleos agrarios y canceló la intervención del gobierno al permitir que, por decisión de la asamblea general del núcleo, se modifique el tipo de propiedad de la tierra. ${ }^{22}$ Estos diversos cambios se combinaron en el nuevo diseño del marco regulatorio sobre impactos sociales.

\section{Usos y costumbres de Pemex y cFe en cuanto a impactos sociales}

Desde su creación, Pemex y la CFE se constituyeron como monopolios de Estado e inscribieron su misión nacional y social como objetivos centrales en sus estatutos jurídicos. ${ }^{23}$ Sin embargo, en la práctica, estos objetivos se cumplieron sólo de manera parcial e insuficiente. ${ }^{24}$

"Al servicio de la nación" -el lema de Pemex durante muchos años- fue un enunciado que sujetó la paraestatal al diktat gubernamental. A lo largo del tiempo y según las necesidades del gobierno, la empresa pasó de ser una palanca para propiciar el desarrollo por sustitución de importacio-

urbanos. Hoy es un órgano administrativo desconcentrado de la Secretaría de Desarrollo Agrario, Territorial y Urbano (Sedatu).

${ }^{22}$ La reforma del campo de 1992 ya consideraba aspectos de derechos humanos, específicamente los vinculados a género. Históricamente, las mujeres no podían ser propietarias de las parcelas. Véase A. Warman, art. cit.

${ }^{23}$ Pemex no puede funcionar sobre la base de la responsabilidad social de empresas porque no es privada: debe dar la cara y someterse a convenios, acuerdos y negociaciones con los ciudadanos para quienes trabaja que, además, legalmente son sus dueños. Rodolfo Uribe Iniesta, "Tendencias y contexto a 37 años del boom petrolero en los llanos costeros del sur del golfo de México”, en Myrna Delfina López Noriega (coord.), Aspectos sociales de la industria petrolera mexicana, Ciudad del Carmen, Universidad Autónoma del Carmen/Consejo Nacional de Ciencia y Tecnología/PyV., 2014.

${ }^{24}$ I. Rousseau, La nueva regulación..., op. cit. 
nes, ofreciendo productos petrolíferos a bajo precio para favorecer el florecimiento de la industria nacional, a convertirse en el factor principal del ajuste macroeconómico del país, a partir de 1978. El caso de la cFE fue similar. Tenía como misión dotar de energía eléctrica a todo el país: una encomienda loable si no fuera porque, muchas veces, se efectuó a costa de poblaciones enteras que fueron desplazadas, sin su consentimiento y sin una reparación de daños adecuada, para construir presas e instalaciones hidroeléctricas.

Durante esos años, no se tomaron en cuenta las exigencias de los grupos sociales afectados por las políticas energéticas. ${ }^{25}$ La preocupación por cumplir con los objetivos políticos y la renta petrolera superó con creces la atención prestada a las externalidades negativas, frecuentemente depredadoras en el caso de la industria petrolera. ${ }^{26}$

Fue solamente a partir de los años noventa que la cuestión social empezó a adquirir relevancia para estas dos empresas, a tono con la preocupación que cundía a nivel internacional por el desarrollo sustentable (es decir, seguridad industrial, responsabilidad social y ambiental). ${ }^{27}$ Sin embargo, aun cuando a nivel interno se tomaron varias medidas para atenuar

${ }^{25}$ Isabelle Rousseau, entrevista con Saúl de la Torre, exgerente de Desarrollo Social de Pemex (2000-2006), 2002. En su opinión, en tiempos del PRI, Pemex negociaba directamente con el gobernador para conseguir el derecho de paso. De ser así, es de pensar que la cFe actuaba de la misma manera.

${ }^{26}$ Véase Armando García Chiang y Josué de Israel Rodríguez Mejía, "Responsabilidad social en la empresa. La región marina noreste de Pemex Exploración y Producción”, Equilibrio Económico (2008), núm. 1, pp.17-40; R. Uribe, op. cit., p. 325. El estado de Tabasco padeció esta situación en los años setenta con el hallazgo de yacimientos y campos petroleros muy prolíficos. Alza inflacionaria desproporcionada, degradación del medio ambiente y del patrimonio arqueológico, corrupción creciente, división social entre los petroleros y el resto de la población, prostitución galopante, alcoholismo fueron algunas de las tristes consecuencias del auge del petróleo allí.

${ }^{27}$ Con el proceso de negociación y la firma del TLCAN, el país tuvo que atender las normas en uso a nivel internacional para proyectar en el exterior una imagen positiva. 
estos problemas, ${ }^{28}$ éstas distaron de propiciar la creación de un arreglo institucional adecuado. En el caso de Pemex, hasta 2014, la Gerencia de Responsabilidad y Desarrollo Social, adscrita a la Dirección General Corporativa de Administración, ${ }^{29}$ condujo bajo un mismo enfoque el diseño e instrumentación de los programas de desarrollo comunitario: el Programa de Apoyo a la Comunidad y Medio Ambiente (PACMA), las Obras de Beneficio Mutuo (OBM) y los donativos y donaciones. ${ }^{30}$

Paralelamente, por ser estratégicas las actividades del sector, conforme a lo dispuesto en los artículos 27 y 28 de la Constitución, la expropiación podía requerirse en caso de necesidad. Y visto que la regularización de la propiedad solía

28 Por ejemplo, la creación de la Gerencia de Desarrollo Social en Pemex (1984) y en la CFE (1989). Por otro lado, abundan los esfuerzos de las dos paraestatales para simular el cumplimiento de los estándares internacionales o nacionales, sin que hayan garantizado una real atención a los reclamos sociales, por ejemplo, el Global Reporting Initiative (GRI) en el caso de Pemex o la certificación nacional Empresa Socialmente Responsable (ESR), otorgada por el Centro Mexicano para la Filantropía (Cemefi), en el de la CFE.

${ }^{29}$ Isabelle Rousseau, entrevista con Jimena Marván, Gerencia de Desarrollo Sustentable y Ambiental de la Subdirección de Operación y Ejecución de la Estrategia, Pemex, 17 de marzo de 2013. Marván explicó que la Gerencia de Desarrollo Social en la Dirección Corporativa de Responsabilidad y Desarrollo Social era un instrumento político cercano al presidente de la República.

${ }^{30}$ Las relaciones de Pemex con la comunidad y sus autoridades estuvieron basadas en los acuerdos marco anuales que la empresa firmaba con los gobiernos de los Estados petroleros. Myrna López Noriega, "Responsabilidad social de Pemex. La evolución en su plan de negocios", en Esther Solano Palacios y Moisés Frutos Cortés (coeds.), El impacto de la reforma energética en México: una mirada nacional y regional, Ciudad del Carmen, Unacar/Amecider, 2016, p. 212. Pemex se comprometía a fomentar la inversión y el empleo, a promover la formación de recursos humanos de alto nivel, a apoyar la realización de infraestructura vial, portuaria e hidráulica, equipamiento urbano y rural y a impulsar proyectos productivos, entre otras acciones. Por otra parte, seguía vigente el viejo sistema -muy oscuro- de donativos y donaciones que Pemex otorgaba. A pesar de ello, los ingresos derivados de la actividad petrolera no dispararon el desarrollo social y económico de los estados petroleros. 
ser un caos, en aras de ganar tiempo, Pemex se acostumbró a negociar de manera poco transparente y dar un pago directo a las autoridades de las comunidades por las afectaciones padecidas. Esto alentó prácticas de reclamación que se tradujeron en extorsiones por parte de "sindicatos" (en particular, en Tabasco), es decir, grupos de presión que cerraban el paso a las compañías contratistas de Pemex para cobrarles un "derecho de piso".

Para paliar todas estas deficiencias, la reforma constitucional de 2013 y sus leyes reglamentarias de 2014 se abocaron a construir una verdadera regulación social de cara a la entrada de actores privados en el sector.

\section{La nueva legislación: características y aportaciones}

Primero se creó en la Sener una Dirección General de Impacto Social y Ocupación Superficial (DGISOs) con las competencias y atribuciones administrativas para ser el órgano ejecutor de la nueva legislación en materia social. ${ }^{31}$ Por su parte, los artículos 118 y 121 de la Ley de Hidrocarburos y los artículos 78 a 89 de su respectivo reglamento vinieron a ser los rectores de la política pública en materia de impacto social y consulta previa a las comunidades.

Se crearon mecanismos de diálogo entre los diversos actores en este entorno de transformación institucional. ${ }^{32}$ Las nuevas herramientas contempladas por dicha normatividad -la Evaluación de Impacto Social, el Plan de Gestión, el Plan de Inversión Social y la Consulta Previa- deben atender y per-

${ }^{31}$ Se pensaba al inicio colocar esta dirección en la Secretaría de Medio Ambiente y Recursos Naturales (Semarnat), puesto que ya estaba a cargo de la Manifestación de Impacto Ambiental. Sin embargo, por la negativa de la Semarnat, fue colocada en la Sener.

32 Katya Puga, consultora y exdirectora general de la DGisos de la Sener, comentarios en el diplomado Impactos Sociales y Consulta Indígena en Energía, Facultad Latinoamericana de Ciencias Sociales (FLACSO), junio de 2018. 
mitir administrar los diversos riesgos ligados a la presencia de infraestructura de los megaproyectos en las comunidades. Para facilitar la negociación entre las partes, se buscó favorecer un diálogo abierto que permitiera tomar en cuenta las percepciones y las diferentes demandas de los actores (stakeholders) con el fin de conseguir acuerdos equitativos, en un contexto en el que deberían prevalecer los "principios de sostenibilidad y respeto de los derechos humanos de las comunidades y pueblos de las regiones en los que se pretendan desarrollar". 33

Las leyes de Hidrocarburos y de Industria Eléctrica plantean dos mecanismos distintos: la Evaluación de Impacto Social y la Consulta Previa, la cual se aplica sólo para comunidades o pueblos indígenas (o tribales). En ambos casos, se propusieron lineamientos, disposiciones y protocolos de acción cuya aplicación debe ser procurada por la DGISOs para evitar que el proyecto sea cancelado. A continuación, se describe a grandes rasgos cada instrumento y se repasan los conceptos clave que definen el ámbito de acción de cada uno, a fin de restituir el significado que se le quiso otorgar.

\section{La Evaluación de Impacto Social (Evis)}

Se trata de un estudio que las empresas deben llevar a cabo y someter a la Sener en aras de conseguir el permiso para desarrollar un determinado proyecto. El reporte correspondiente debe permitir una estimación de las posibles repercusiones vinculadas a la implantación y ejecución del proyecto; también debería ser un medio idóneo para entablar relaciones con la comunidad. ${ }^{34}$ Además, la necesidad de realizar una consulta previa se desprende de la Evis, en caso de que haya comunidades indígenas cerca del área del proyecto. Se han dado situaciones en que la autoridad ha determinado

${ }^{33}$ Loc. cit. Las cursivas son del autor.

${ }^{34}$ No siempre es el caso. 
procedimientos de consulta sin mediar una EVIs, basándose sólo en sus propios análisis técnicos. ${ }^{35} \mathrm{El}$ desarrollador (empresa estatal o privada) que lleva a cabo la Evis puede determinar a quién consultará, aunque de manera casi automática debe integrar las características geográficas, demográficas, culturales, económicas y sociales de comunidades y municipios. Paralelamente, realiza un mapeo de actores (y sus agendas múltiples) para visualizar los impactos y, sobre todo, la reacción de los posibles afectados. Esto ayuda a la empresa a prever y negociar con la comunidad las medidas de prevención, mitigación o reparación de los daños. Desafortunadamente, las empresas suelen considerar la Evis como una simple formalidad, cuando debería ser vista como un primer contacto de "buena vecindad" entre la empresa y la comunidad, dos actores que, probablemente, van a cohabitar durante más de veinte años. ${ }^{36}$

La escala que permite caracterizar las repercusiones, desde poco graves hasta significativas, es decir, que podrían poner en peligro la subsistencia física y cultural de la comunidad, se remite al principio "precautorio" o de "precaución". 37

La Evis está basada en un marco normativo; leyes, normas y protocolos definen la metodología que debe seguirse para evaluar la situación que priva en el área (identificando la línea base del tiempo, las áreas de influencia, la zona de amor-

35 Fue el caso del Parque Eólico del Sur, en Juchitán de Zaragoza, Oaxaca, o el del Gasoducto Sonora, con los yaquis. E. Castro, supra.

36 Muchos de los consultores que realizan las Evis a nombre de sus clientes señalan que cuando la empresa toma este proceso con seriedad es más difícil que surjan luego conflictos con la comunidad.

${ }^{37}$ En el caso de Monsanto, la Suprema Corte de Justicia de la Nación (SCJN) lo definió en estos términos: "Surge como respuesta ante la falta de certeza científica sobre los posibles efectos ambientales de determinadas actividades. Por ello, este principio requiere adoptar disposiciones de modo restrictivo, tomando medidas para que ese daño hipotético no llegué a producirse". Véase Héctor Garza, "Los aspectos sociales del sector energético", presentación en el curso Actualización de los Sistemas Energéticos, Consejo Mundial de Energía / Sener / IneEL / El Colegio de México, Ciudad de México, 20 de septiembre de 2016. 
tiguación, etc.), ${ }^{38}$ así como los criterios para elaborar un plan de mitigación y compensación. En cuanto al Plan de Gestión Social, producto de la Evis, las Disposiciones Administrativas lo definen como "una estrategia de implementación de medidas positivas y de reparación”. Con el plan, se busca administrar la vida útil del proyecto para atender, en cada una de sus etapas, los impactos diferenciados que podrían presentarse, desde la preparación del sitio hasta su abandono. Indudablemente representa también un marco útil para controlar las actividades futuras de las empresas. Paralelamente, el Plan de Inversión Social está destinado a mitigar los impactos que se presentarán. Debe fundamentarse de manera sólida para poder diferenciar la inversión que por esencia le corresponde al gobierno, de la que le corresponde al promovente. ${ }^{39}$

Existe otro reporte, denominado Estudio de Impactos Sociales, diferente de la Evaluación de Impactos Sociales y que concierne solamente a la exploración y explotación de los hidrocarburos. Le corresponde a la Sener realizarlo y presentarlo antes de cualquier licitación. Es un análisis muy útil que permite a las compañías estimar mejor las contraprestaciones que pueden ofrecer en la licitación. ${ }^{40}$ Basada en el principio de sustentabilidad y una perspectiva de derechos humanos, la Evis es una novedad en México y sienta un precedente que debería aplicarse en otros sectores. ${ }^{41}$

${ }^{38}$ La línea base debe fijarse; va a ser el criterio para precisar las alteraciones futuras.

${ }^{39}$ Frecuentemente se ha señalado que el gobierno busca cargar a la empresa con inversiones que de hecho le corresponden a él; esto, en detrimento de la comunidad, que pierde así recursos.

40 Este Estudio de Impactos Sociales se aplica solamente para los hidrocarburos. Según Julia Puente, fue un olvido del legislador, puesto que no se aplica a la industria eléctrica. Dirección General de Impacto Social y Ocupación Superficial, DGISos, Taller de Responsabilidad Social Corporativa ante la Reforma Energética, Programa de Energía / Social License / Conecta Cultura, El Colegio de México, 9 de noviembre de 2016.

${ }^{41}$ En realidad, el sector minero también lo adoptó. 
La Consulta Previa, Libre e Informada, y de buena fe

Es obligatorio realizar una Consulta Previa, Libre e Informada (CPLI) cuando el proyecto va a desarrollarse en los alrededores de una o varias comunidades o pueblos indígenas o tribales. En esta circunstancia, la Sener desempeña un papel fundamental: organiza el proceso y es autoridad competente para emitir el acto definitivo. Sin embargo, en última instancia, son las comunidades indígenas las que deben resolver si aceptan o no la presencia del proyecto. Las autoridades de la comunidad indígena marcan los tiempos, el ritmo y las modalidades del proceso, que deben ajustarse a sus costumbres y su agenda (como fiestas y ceremonias). La empresa, por su lado, no puede asistir para no influir en los resultados.

$\mathrm{Al}$ promover la participación de los indígenas en la toma de decisiones, el derecho a la cPLI les ofrece una dimensión social y política: permite garantizar sus derechos y su permanencia física y cultural. ${ }^{42}$ También reconoce la diversidad étnica y cultural de México, y subraya la importancia del diálogo y de la negociación con respeto y corresponsabilidad, privilegiando el consenso y el consentimiento.

La CPLI debe identificar los impactos que el proyecto podría tener en la comunidad para determinar las acciones necesarias en aras de prevenir o remediar las repercusiones, y busca los posibles precedentes o los documentos previos aceptablemente confiables. Para tener validez, la cPLI debe ser "previa, libre (sin coacción), informada, pertinente culturalmente (con traductor en la lengua materna) y de buena fe”. Además, sólo el indígena (u otro miembro de una nación tribal) es sujeto de derecho a la consulta.

Ambos instrumentos, EVIS y CPLI, buscan promover el diálogo y la negociación, multinivel y multicultural, entre los diversos actores. ¿Quiénes son los actores involucrados, directa o indirectamente, en estos dos ámbitos de negociación?

${ }^{42}$ En este sentido, ya no son solamente receptores. 


\section{Una gestión multiactores}

El nuevo marco regulatorio toma en cuenta la presencia de actores múltiples con agendas muy diferenciadas, incluso hasta antagónicas. Y de ambos procesos mencionados, la CPLI ofrece el escenario más complejo.

La DGIsos adoptó como modalidad formar un comité con diversos miembros, los cuales suelen diferir según los casos. ${ }^{43}$ Las dependencias dedicadas a la atención de la población indígena, en particular la CDI, por principio deberían involucrase de manera casi automática, lo cual no siempre es el caso. Al respecto, la CDI alega su falta de personal. La Secretaría de Gobernación (Segob) por ley debe ser partícipe, ya que se trata de un tema de gobernabilidad, aunque no siempre lo hace. Es notable que quien fuera el segundo director general de Impactos Sociales y Ocupación del Suelo, en el periodo 2016-2018, Rodolfo Salazar, proviniera de la Segob; ${ }^{44}$ esto subraya el vínculo entre ésta y la Sener, dado que los temas de derechos humanos e indígenas pertenecen al campo de acción de Gobernación.

Uno de los grandes retos de la CPLI -y, en general, del marco regulatorio- es definir el actor colectivo indígena, por encima del individual, es decir, la comunidad o pueblo. La dificultad es doble: aclarar en cada caso la presencia tanto del "indígena" como del "actor colectivo". De acuerdo con el artículo 2 de la Constitución, "la conciencia de su identidad indígena deberá ser criterio fundamental para determinar a quiénes se aplican las disposiciones sobre pueblos indígenas". Los pueblos y las personas indígenas tienen el derecho y la responsabilidad de definir su pertenencia a

43 No siempre se formó un comité. Fue el caso de la consulta a la etnia yaqui sobre el gasoducto Sonora. Se requirió celebrar la consulta únicamente con Sener.

44 Fue, primero, subdirector de Atención a Defensores, en la Unidad para la Defensa de los Derechos Humanos (agosto de 2013-junio de 2014) y luego director de Atención a Casos, en la misma unidad (julio de 2014-agosto de 2015). 
estas colectividades, lo que despoja al Estado de esta prerrogativa. Por su lado, el Convenio 169 de la orT afirma que los propios indígenas tienen el derecho de autoidentificarse como tales. ${ }^{45}$ Además, es preciso identificar al sujeto colectivo -el pueblo constituido y las comunidades indígenas (o afrodescendientes o equiparables)-, puesto que la legislación indica que los individuos como tales no están autorizados a participar. ${ }^{46}$

La norma excluye de la CPLI a quienes son ajenos a la comunidad: las entidades productivas, las organizaciones sociales, los sindicatos, etc. Por su lado, los gobiernos estatales y municipales, los organismos de derechos humanos y las asociaciones o instituciones de la sociedad civil, en calidad de observadores, pueden facilitar el proceso. Cada caso es distinto y los participantes varían mucho. ${ }^{47}$

Finalmente, las instituciones financieras internacionales son un actor fundamental, aunque poco visible. Vinculadas con el desarrollador del proyecto, observan con minucia el proceso para decidir si aceptan otorgar el préstamo bancario a la empresa y dar certeza a la inversión.

Indudablemente, este nuevo marco legal sobre impacto social ofrece piezas nuevas en un andamiaje institucional renovado con la reforma energética. Sin embargo, desde el inicio de su implementación, no ha logrado ofrecer los resultados que esperaba el equipo que lo diseñó. Muchos proyectos siguen detenidos y se siguen presenciando muchos conflictos. Uno de los más violentos fue el caso de la matanza del 21 de

${ }^{45}$ H. Garza, op. cit., p. 32.

${ }^{46}$ Según el Centro Profesional Indígena de Asesoría, Defensa y Traducción, Situación normativa de los derechos indígenas en México y experiencias de otros países, Oaxaca, CDI, 2006 , los elementos que deben reunirse para considerar un pueblo como indígena son: tener una unidad de cultura, asumir su pasado histórico, proyectar un futuro común, reconocerse en un conjunto dentro de una identidad colectiva, decidir aceptarse como miembros de esa entidad y referirse a un territorio propio.

${ }^{47}$ En general, en estos procesos, el gobierno municipal tiende a participar de manera mucho más activa que el estatal. 
octubre de 2016 en Loma de Bácum, Sonora, que dejó tres muertos y dos heridos por el enfrentamiento entre miembros de la etnia yaqui a raíz del paso del gasoducto de IEnova por sus tierras. Por su lado, ATCo - una compañía canadiense de gasoductos- no ha podido finalizar la construcción de uno, tendido sobre 17 kilómetros, a pesar de haber ganado el proyecto (construcción, mantenimiento y operación) el 13 de febrero de 2015. Hoy en día, incluyendo todos los sectores, de los 400 megaproyectos de infraestructura cuyo desarrollo está en situación de conflictividad social, más de 70, casi una cuarta parte, pertenecen al sector energético. Es una circunstancia interesante porque los demás sectores no están dotados de una legislación tan ambiciosa como la que tiene éste.

¿Por qué esta nueva legislación no ha logrado disminuir de manera notable los riesgos de conflicto? ¿Cuáles son sus lagunas o las piezas faltantes? ¿Qué retos no fueron abordados en el momento del diseño institucional, lo que hubiera permitido una mejor gobernabilidad tanto sectorial como local, regional y nacional?

\section{UNA LEGISLACIÓN INCOMPLETA Y LIMITADA}

Son varios los problemas ligados a la construcción y la implementación del nuevo marco regulatorio. En buena parte se debe a los mecanismos elegidos para armar el andamiaje institucional necesario: sus posibles indefiniciones, sus incoherencias y las lagunas que subsisten. Más graves son los aspectos de tipo estructural.

\section{En relación con la reforma energética}

De manera similar a la puesta en marcha del resto de la reforma energética, la nueva legislación social tuvo que enfrentar dos retos de envergadura: el timing con el que se implementó 
y la curva de aprendizaje de los diferentes actores, muy abrupta, porque se partía de cero.

El timing fue un elemento crítico en la reforma al colocar a todos los actores nacionales del sector en una situación totalmente nueva: debían aprender sobre la marcha. La ejecución del proyecto se realizó con una premura poco indicada, en un momento en el que, a nivel internacional, se vivía un contexto sumamente complejo por la drástica caída de los precios del crudo (76\% entre 2015 y 2016). Lejos de ser parte de un cálculo de optimización económica, como lo pretendía el discurso oficial, este timing forzado respondía a un cálculo político-electoral. En cuanto a la nueva regulación social, no todos los grandes actores comprendían las nuevas reglas de la misma manera. Esto complicó la posibilidad de un diálogo sereno para lograr una decisión consensuada. De los tres principales grupos de intereses, sólo los desarrolladores -en particular las compañías extranjerasconocían y manejaban las reglas del juego, por su experiencia en los diferentes países en donde operan, sin contar con que muchas poseen reglamentos internos de responsabilidad social y saben adecuarse a los requisitos legales para conseguir una licencia social sin afectar la rentabilidad del proyecto. El gobierno, al forjar las reglas del juego y ser el mediador final, ocupó también una posición ventajosa. Sólo las comunidades desconocían estas reglas, además de que, en su mayoría, sólo tienen una visión local. Esto las coloca en una posición de inferioridad: falta de información, problemas de traducción y de comprensión de la lógica del juego. Por carecer de información, están frecuentemente sujetas a rumores y, por ende, a manipulación. Este desequilibrio entre los tres grupos implica que el Estado deba asegurar el respeto y la protección de los derechos humanos cuando los pueblos indígenas se sienten a la mesa para negociar.

La recién creada DGISOs, por su parte, presenta una situación problemática en diversos aspectos. Para empezar, su ubicación en la Sener no es adecuada: carece de autonomía y es juez y parte a la vez. Por naturaleza, el secretario de Ener- 
gía responde a una lógica sexenal (en lo político-electoral y en términos de gobernabilidad), mientras que los proyectos de infraestructura son de largo plazo (de veinte a treinta años). Además, no tiene plena capacidad de acción. Asimismo, las carencias presupuestales han limitado el alcance de los resultados: muy poco personal para la cantidad de Evis a revisar y escasos recursos económicos para dar seguimiento a los planes de gestión. ${ }^{48}$ Esto contrasta con Colombia: para las mismas funciones, la entidad correspondiente tiene 250 empleados, al tiempo que en México sólo se cuenta con 15). ${ }^{49}$ De la misma manera, las atribuciones administrativas de la DGISOs son débiles: no le fue otorgada una facultad sancionadora en caso de que la empresa no presente su Plan de Gestión Social. Tampoco tiene la de monitorear el cumplimiento de este plan ni de la Evis.

Por otra parte, la legislación sobre Evis y CPLI presenta deficiencias. Las Disposiciones Administrativas de Carácter General sobre las Evis -documento rector oficial- se publicaron en el Diario Oficial de la Federación el 1 de junio de 2018. ${ }^{50}$ Falta, por otro lado, definir claramente qué proyectos necesitan Evis, así como diferenciar su complejidad (estaciones de servicio, gasoductos, pozos de exploración, desarrollo o de producción) que ameritaría Evis diferenciadas. El Estudio de Impacto Social, que sólo está previsto para el subsector hidrocarburos, debería extenderse al subsector

${ }^{48} \mathrm{Al}$ ser un tema tan complejo y al recaer las demandas sociales de las comunidades en diferentes ámbitos, como el agrícola, de tenencia de la tierra, el laboral, el ambiental, de salud, sería pertinente un mayor trabajo interinstitucional; por ejemplo, crear una comisión intersecretarial de impacto social de proyectos de infraestructura, similar a las que existen en otros temas, como cambio climático. Permitiría diagnosticar desde una visión sistémica los conflictos y proponer soluciones integrales.

${ }^{49} \mathrm{~J}$. Puente, supra.

${ }^{50}$ Acuerdo por el que se emiten las Disposiciones Administrativas de Carácter General sobre la Evaluación de Impacto Social en el Sector Energético, Diario Oficial de la Federación, 1 de junio de 2018, https:// www.dof.gob.mx/nota_detalle.php?codigo=5524885\&fecha=01 $/ 06 / 2018$ 
eléctrico, que enfrenta complejos y crecientes conflictos. Finalmente, debe vincularse el tema ambiental al problema social y, por ende, la MIA con la Evis.

Igualmente, hay que buscar caminos para vincular la EvIS con la CPLI, ya que son mecanismos que van en la misma dirección. Esta última, por su lado, carece de una legislación propia y adecuada, lo cual conduce a situaciones caóticas y pone en riesgo tanto las inversiones como el derecho de las comunidades. ${ }^{51}$ La pregunta sobre la localización del organismo que se responsabilice de las consultas -cuestión constante durante el sexenio de Peña Nieto- debe encontrar respuesta. La brecha entre el marco legal de derechos humanos y su implementación, sobre todo en el caso de la CPLI, amerita plena atención, así como el desfase temporal entre la consulta y la licitación. ${ }^{52}$ Finalmente, la falta de contacto vulnera el derecho de información de las comunidades, ya que no les permite saber sobre la tecnología, los beneficios y los riesgos del proyecto.

Para implementar la regulación, es necesario que se coordinen los tres niveles de gobierno (federal, estatal y municipal), lo cual raras veces se está dando, pues cada ente responde a intereses políticos diferentes, por no decir contrarios. Por su lado, en la gestión local, los legisladores desempeñan un papel fundamental, dado que la política no es ajena al sector energético. Las demandas sociales que surgen a raíz de proyectos energéticos suelen mezclarse con rezagos sociales e intereses político-electorales. Una adecuada normatividad y su debida aplicación organizativa y operativa deberían ayudar a transparentar las acciones ante las demandas y los conflictos sociales, así como disminuir que se recurra al juego político-electoral.

${ }^{51}$ José Alberto Moreno Chávez, "El régimen internacional de derechos humanos y su implicación para el caso de las consultas en proyectos energéticos en México. Un acercamiento teórico a la problemática”, Foro Energético, núm. 5, 2017.

${ }^{52}$ La CPLI constituye un requisito de la licitación. 
En lo que respecta a los actores, las dificultades son diferentes. En relación con las empresas, las medianas y pequeñas del país manifiestan un gran desconcierto respecto a la nueva regulación social. Ante su falta de experiencia, se crearon -frecuentemente al vapor- muchas consultoras para realizar EVIs, carentes de conocimientos en temas de derechos humanos, indígenas y de experiencia con las comunidades, de modo que desde el inicio ha privado una ausencia de profesionalización de los consultores en este campo. ${ }^{53}$

En cuanto a los grupos comunitarios, sobre todo indígenas, su cosmovisión es radicalmente distinta a la de los demás actores. Los pueblos indígenas tienen sus propios dialectos que encierran referencias muy específicas y frecuentemente diametralmente opuestas a las que predominan en la sociedad actual. La noción del tiempo lo ilustra perfectamente. Mientras que para un inversionista el tiempo es dinero e implica extremar la velocidad en la construcción del proyecto, para un indígena representa la oportunidad de forjar relaciones y ganar confianza con el interlocutor. ${ }^{54}$

Además, el nuevo entorno organizacional no facilita la fluidez en la comunicación entre múltiples actores. Las reglas padecen de cierta laxitud en sus definiciones. Ése es el caso del sujeto "indígena”: ¿`según qué criterios valorar la autoproclamación de la identidad individual cuando la persona vive fuera de su comunidad desde hace muchos años y ha cambiado su forma de vida tradicional? La subjetividad que la acompaña puede resultar problemática. Siguen prevaleciendo diversos criterios para el reconocimiento de la población indígena. Frente a ello, es imprescindible que se haga un registro de las comunidades originarias, como sucedió en países como Perú. 55

Por su lado, el concepto de "previo" en la CPLI no es del todo claro: ¿previo a qué? Estas dificultades remiten a un pro-

${ }^{53}$ Se están remediando poco a poco estas lagunas.

${ }^{54}$ Otro ejemplo: los indígenas de Oaxaca tampoco entienden que el viento, elemento natural por excelencia, tenga precio en el mercado.

${ }^{55}$ K. Puga, op. cit. 
blema teórico-metodológico que suele lleva a la judicialización de los casos. La noción de "impacto significativo" es del mismo calibre: reenvía a un principio precautorio (la simple susceptibilidad del impacto) que surge como respuesta ante la falta de certeza científica sobre los posibles efectos ambientales o sociales de determinadas actividades. ${ }^{56}$ La inconformidad ante los procesos de CPLI ha ocasionado la judicialización de los proyectos. Sin duda, éste es el tema más complejo: proviene de una reforma constitucional en materia indígena que acarreaba muchas inconformidades cuando se aprobó en 2001 (en el marco del proceso de paz y concordia para Chiapas). ${ }^{57}$ Sería adecuado definir los diferentes conceptos ("previo" y otros) apegándose a los estándares internacionales, revisando la jurisprudencia de la Corte IDH, los informes de comités, grupos de trabajo, y relatores de Naciones Unidas, así como las mejores prácticas en otros países. ${ }^{58}$

Finalmente, no se ha discutido con la profundidad que lo amerita la inseguridad en el país y su relación con los conflictos sociales, secuestros, amenazas, derecho de piso, extorsión. ¿Qué relación hay entre problemas de inseguridad y conflictos sociales por la presencia de un megaproyecto en cierta zona? ¿Cómo van a manejar esta situación las empre-

56 H. Garza, op. cit.

${ }^{57}$ K. Puga, op. cit. Por su parte, Rodolfo Stavenhagen estimó que los Acuerdos de San Andrés, firmados entre el EzLN y el gobierno federal en 1996, indicaron la vía de una salida política al conflicto, que derivó en la iniciativa legislativa. Naciones Unidas, Consejo de Derechos Humanos, Informe del relator especial sobre la situación de los derechos humanos y las libertades fundamentales de los indígenas, Rodolfo Stavenhagen, misión a México, 23 de diciembre de 2003, UN Doc. E/CN.4/2004/80/Add.2, p. 2.

58 Naciones Unidas, Consejo de Derechos Humanos, Informe del relator especial sobre la situación de los derechos humanos y libertades fundamentales de los indígenas, James Anaya, UN Doc. A/HRC/12/34, 15 de julio de 2009, párr.65; Olivier de Schutter, Informe de misión a México, México, Oficina del Alto Comisionado para los Derechos Humanos, 2012, párr. 40. Corte IDH, Saramaka vs. Suriname. Excepciones preliminares, Fondo, Reparaciones y Costas, sentencia del 28 de noviembre de 2007, Serie C, núm. 172. 
sas? ¿Cuál ha sido la experiencia de un país como Colombia? ${ }^{59}$ ¿Cómo evitar que un proyecto forme parte del círculo de las extorsiones y se vuelva rehén de situaciones que ahondan los conflictos en las comunidades? 60

\section{Los problemas estructurales}

La reforma energética nació en el contexto de un proyecto económico de índole neoliberal. La nueva regulación se sitúa en el cruce de dos visiones antagónicas del país: por un lado, el viejo régimen posrevolucionario de corte popular y nacionalista, edificado sobre el poder del Estado, versus un proyecto tecnocrático y liberal basado en los valores del mercado. La reconciliación es sumamente compleja y los actores están muy divididos. A muchos campesinos, la Revolución les hizo justicia al devolverles tierras acaparadas por los latifundistas y suelen ver a los nuevos inversionistas como nuevos expoliadores, cuando no como nuevos conquistadores. Por otro lado, a pesar del discurso nacionalista revolucionario, las desigualdades y el rezago del campo han sido una realidad constante, de modo que en el plano social, la nueva legislación y la construcción institucional tienen que hacerse cargo de la deuda histórica de la nación con los pueblos indígenas y el campo, en general. Es un reto muy complejo por no decir casi imposible. La reforma también surgió en medio de una transición democrática que sigue inacabada. Los mecanismos tradicionales de control ya no están vigentes, pero no han sido reemplazados por instrumentos más acordes con el nuevo sistema político democrático. Esto propició

${ }^{59}$ Diagnóstico de la conflictividad social: estrategia territorial para la gestión equitativa y sostenible del sector hidrocarburos, Bogotá, Agencia Nacional de Hidrocarburos / Ministerio de Minas y Energía / Ministerio del Interior / Ecopetrol / PNUD, 2016, http:/ /www.co.undp.org/content/dam/colombia/docs/DesarrolloHumano/Diagn\%C3\%B3stico\%20de\%20la\%20conflictividad\%20social.pdf

${ }^{60}$ K. Puga, op. cit. 
grandes vacíos de poder y de autoridad mientras que, por otro lado, la construcción de la sociedad civil no ha logrado adquirir plena fuerza. En un contexto institucional debilitado, este antagonismo socio-histórico no resuelto tiene repercusiones en el desarrollo de los nuevos proyectos de infraestructura. La presencia de sus instalaciones en comunidades muy sensibles a la injerencia externa polariza estas carencias de gobernabilidad. Por sí sola, la nueva regulación para la gestión de los impactos sociales no puede remediar conflictos que emanan de otros ámbitos. De manera implícita, se ha atribuido a la nueva legislación y a la DGISos la misión de fortalecer la gobernabilidad del país, tarea para la que no está preparada, pues no tiene las herramientas ni la capacidad para hacerlo. Esto resulta de una confusión entre legalidad y legitimidad. Como tal, la nueva legislación tiene a bien cumplir con criterios de legalidad, pero esto no le brinda la legitimidad suficiente ante los ojos de la población -en particular, las comunidades rezagadas- para que la acepten y la acaten. Entonces, está dotada de una legalidad que carece de legitimidad.

\section{REFLEXIONES FINALES}

El problema fundamental al que se enfrenta el Estado mexicano es resolver la tensión entre dos misiones: garantizar la seguridad energética (con la infraestructura necesaria para responder a la demanda) y, al mismo tiempo, respetar y proteger los derechos de las comunidades y las poblaciones donde se desarrollan los proyectos de energía. En otros términos, hay que equilibrar los objetivos económicos con base en inversiones rentables, y la seguridad energética a mediano y largo plazo, con la gobernabilidad. ${ }^{61}$ Los nuevos mecanismos de regulación y gestión social de los megaproyectos que instauró la reforma energética debían crear una garantía

${ }^{61}$ I. Rousseau, I., La nueva regulación..., op. cit., p. 16. 
institucional para anticiparse al conflicto y llegar a un entendimiento. En teoría, las EVIS y CPLI bien elaboradas y ejecutadas, con el cumplimiento de los estándares mínimos en materia de derechos humanos, otorgarían la "licencia social" para operar. Hasta ahora no ha sido así, los conflictos no han disminuido y no se ha logrado un mayor entendimiento entre las partes.

Si bien no será muy complicado atender y remediar en el futuro las deficiencias legales y normativas anteriormente señaladas, en cambio, el problema de fondo será atender la parte estructural del problema, es decir, legitimar estos instrumentos. Ello implica que el Estado mexicano enfrente con seriedad las carencias sociales que olvidó atender desde hace siglos: desigualdad profunda, discriminación hacia ciertos sectores de la población (pueblos indígenas y otros pueblos tribales), un Estado de derecho débil (corroído por una corrupción demasiado expandida) y una violencia que, de alguna manera, se vincula con los aspectos anteriores.

Sólo así la nueva legislación adquirirá plena fuerza y vigencia y podrá cumplir con los objetivos esenciales en materia energética: la seguridad y la sostenibilidad energéticas pero también -y no es un asunto menor- el problema general de la gobernabilidad.

\section{REFERENCIAS BIBLIOGRÁFICAS}

Acuerdo por el que se emiten las Disposiciones Administrativas de Carácter General sobre la Evaluación de Impacto Social en el Sector Energético, Diario Oficial de la Federación, 1 de junio de 2018, https://www.dof.gob.mx/nota_detalle.php?codigo= $5524885 \&$ fecha $=01 / 06 / 2018$

Centro Profesional Indígena de Asesoría Defensa y Traducción, Situación normativa de los derechos indígenas en México y experiencias de otros países, Oaxaca, CDI, 2006. 
Comisión Federal de Electricidad, CFE, "Informe anual 2008", México, http://app.cfe.gob.mx/informe2008/capitulo7_10.html (consulta del 10 de febrero de 2017).

Convenio sobre pueblos indígenas y tribales, 1989 (núm. 169), https://www.ilo.org/dyn/normlex/es/f?p=NORMLEXPUB: 12100:0::NO:12100:P12100_INSTRUMENT_ID:312314:NO

Corte IDH, Saramaka vs. Suriname. Excepciones preliminares, Fondo, Reparaciones y Costas, Sentencia del 28 de noviembre de 2007, Serie C núm. 172.

"Decreto por el que se reforman y adicionan diversas disposiciones de la Constitución Política de los Estados Unidos Mexicanos, en Materia de Energía", Diario Oficial de la Federación, 22 de diciembre de 2013, http://www.dof.gob.mx/nota_detalle.php? codigo $=5327463 \&$ fecha $=20 / 12 / 2013$

"Diagnóstico de la conflictividad social: estrategia territorial para la gestión equitativa y sostenible del sector hidrocarburos”, Bogotá, Agencia Nacional de Hidrocarburos / Ministerio de Minas y Energía / Ministerio del Interior / Ecopetrol / PNUD, 2016, http:/ /www.co.undp.org/content/dam/colombia/docs/DesarrolloHumano/Diagn \%C3\%B3stico\%20de\%20la\%20conflictividad\%20social.pdf

Diario de los Debates de la Cámara de Senadores del Congreso de los Estados Unidos Mexicanos, LXII Legislatura, año II, tercer periodo extraordinario, vols. II, III, IV y IV.

García Chiang, Armando y Josué de Israel Rodríguez Mejía, "Responsabilidad social en la empresa. La Región Marina Noreste de Pemex Exploración y Producción”, Equilibrio Económico, 2008, núm.1, pp.17-40.

Garza, Héctor, "Los aspectos sociales del sector energético", presentación en el curso Actualización de los Sistemas Energéticos, México, Consejo Mundial de Energía / Sener / InEEL / El Colegio de México, 20 de septiembre de 2016.

Grunstein Dickter, Miriam, "Contra el viento: regulación, crisis social y cambio institucional en el corredor eólico del Istmo”, Economía, Sociedad y Territorio,16 (2016), pp. 485-517. 
Gutiérrez del Ángel, Arturo, La peregrinación a Wirikuta: el gran rito de paso de los huicholes. Etnografía de los pueblos indígenas de México, México, inAH / Universidad de Guadalajara, 2002.

Jobert, Bruno, Les politiques publiques, París, PUF, 1991.

Ley de la Comisión Federal de Electricidad, 2014, Diario Oficial de la Federación, 11 de agosto de 2014, https://www.dof.gob. $\mathrm{mx} /$ nota_detalle.php?codigo=5355990\&fecha=11/08/2014

Ley de Petróleos Mexicanos, 2014, Diario Oficial de la Federación, 11 de agosto de 2014, https://www.dof.gob.mx/nota_detalle. php?codigo $=5355990 \&$ fecha $=11 / 08 / 2014$

López Noriega, Myrna, "Responsabilidad social de Pemex. La evolución en su plan de negocios”, en Esther Solano Palacios y Moisés Frutos Cortés (coeds.) El impacto de la reforma energética en México: una mirada nacional y regional, Ciudad del Carmen, Unacar/Amecider, 2016.

Moreno CHÁvez, José Alberto (2017), "El régimen internacional de derechos humanos y su implicación para el caso de las consultas en proyectos energéticos en México. Un acercamiento teórico a la problemática”, Foro Energético (2017), núm. 5, CEI, El Colegio de México, 2017.

Naciones Unidas, Consejo de Derechos Humanos, Informe del relator especial sobre la situación de los derechos humanos y libertades fundamentales de los indígenas, James Anaya, UN Doc. A/HRC/12/34, 15 de julio de 2009.

Naciones Unidas, Consejo de Derechos Humanos, Informe del relator especial sobre la situación de los derechos humanos y las libertades fundamentales de los indigenas, Rodolfo Stavenhagen, misión a México, UN Doc. E/CN.4/2004/80/Add.2, 23 de diciembre de 2003.

Naciones Unidas, Objetivos de Desarrollo Sostenible, 2015, https:/ / www.un.org/sustainabledevelopment/es/objetivos-de-desarro llo-sostenible/

Nahmad-Sitton, Salomon, El impacto social del uso del recurso eólico, Oaxaca, conAcyt / Centro de Investigaciones y Estudios Superiores en Antropología Social, 2011.

Pirker, Kristina, José Manuel Arias y Hugo Ireta, El acceso a la información para la contraloría social: El caso de las donaciones y donativos de Pemex a Tabasco, México, Fundar, 2007. 
PugA, Katya, consultora y exdirectora general de la DGisos de la Sener, comentarios en el diplomado Impactos Sociales y Consulta Indígena en Energía, Facultad Latinoamericana de Ciencias Sociales (FLACSO), junio de 2018.

Reglamento Interior de la Secretaría de Energía, Diario Oficial de la Federación, 31 de octubre de 2014.

Rousseau, Isabelle, "La nueva regulación de la gestión social de los proyectos energéticos en México, seguridad, sustentabilidad y gobernabilidad", Revista Mexicana de Ciencias Políticas y Sociales, núm. 230 (2017), pp. 197-220.

Rousseau, Isabelle, México: ¿Una revolución silenciosa? 1970-1995. Élites gubernamentales y proyecto de modernización, México, El Colegio de México, 2001.

Schutter, Olivier de, Informe de misión a México, México, Oficina del Alto Comisionado para los Derechos Humanos, México, 2012.

Uribe InIESTA, Rodolfo, Tendencias y contexto a 37 años del boom petrolero en los llanos costeros del sur del golfo de México, Centro Regional de Investigaciones Multidisciplinarias, 2012.

WARMAN, Arturo, "La reforma agraria mexicana: una visión de largo plazo", Land Reform/Réforme agraire/Reforma Agraria, núm. 2 (2003), pp. 84-94.

Zenteno, Javier, Guillermo Pineda y Daniela Robledo, "Impacto social y ocupación temporal”, Energía a Debate (2016), núm. 74.

\section{Otras fuentes}

Rousseau, Isabelle, entrevista con Héctor Garza, 30 de enero de 2017.

Rousseau, Isabelle, entrevista con Saúl de la Torre, exgerente de Desarrollo Social de Pemex (2000-2006), 2002.

Rousseau, Isabelle, entrevista con Jimena Marván, Gerencia de Desarrollo Sustentable y Ambiental de la Subdirección de Operación y Ejecución de la Estrategia, Pemex, 17 de marzo de 2013. 
